\title{
Gobierno abierto y transparencia: construyendo un marco conceptual
}

\author{
Open government and transparency: building a conceptual framework
}

\author{
Rodrigo Sandoval-Almazán / rsandovala@uaemex.mx \\ Universidad Autónoma del Estado de México, México
}

\begin{abstract}
The idea of open government has spread across Latin American countries causing confusion and ambiguous interpretations. The concepts of "open government" and "transparency" have been used as synonyms and have created false expectations, but mostly they have been used to implement public policies and government strategies to accomplish the goal of creating a more open government. The purpose of this research is to provide some theoretical elements to clear this confusion. In order to achieve such goal, related papers of academic journals over the past six years have been reviewed and classified on three main paths of knowledge. From this point a concept of open government is proposed and discussed. The aim of this paper is to contribute with a theoretical framework that supports research on open government, transparency and accountability.
\end{abstract}

Key words: open government, transparency, theory, concepts, e-government.

Resumen: La confusión del término anglosajón open government así como del término transparency han traído consigo ambigüedades y creado falsas expectativas. Además de utilizar ambos conceptos como sinónimos, se han implementado políticas públicas para hacerlos realidad en distintos países latinoamericanos sin tener una dirección precisa o un objetivo claro. Esta investigación busca aportar elementos teóricos que ayuden a delimitar los principales caminos teóricos que se han seguido y proponer un concepto que distinga tanto la transparencia como el gobierno abierto. Para lograrlo se han revisado las principales revistas académicas y artículos de los últimos seis años y se han agrupado en tres corrientes de pensamiento para proponer un concepto sobre gobierno abierto. El objetivo es contribuir a la construcción de una teoría general más sólida, que ayude a entender mejor el gobierno abierto, la transparencia y la rendición de cuentas.

Palabras clave: transparencia, gobierno abierto, teoría, conceptos, e-gobierno. 


\section{Introducción}

El gobierno abierto existe desde el siglo XVI cuando fue establecido en Suecia y los datos gubernamentales fueron hechos públicos como un derecho constitucional; así, la gente pudo tener acceso a esta información (Tauberer, 2012). Parks (1957) escribió un artículo en la revista de Derecho de George Washington, que se tituló: "The Open Government principle: applying the right to know under the constitution", donde comienza su argumentación señalando que tanto el partido demócrata como el republicano habrían prometido impulsar una libertad de información del gobierno de Estados Unidos, pero que no se había cumplido. Esta solicitud es una reacción al hecho de que se ocultara información militar durante la Segunda Guerra Mundial y constituyó el punto de arranque para comenzar a definir lo que es un gobierno abierto.

En los años setenta, la frase del gobierno abierto se vinculó con el secreto gubernamental y el derecho a saber de las decisiones públicas en el Reino Unido (Ivester, 1977; Relyea, 1977). Siguiendo este debate conceptual, Bennett (1985) publicó en Inglaterra un artículo discutiendo sobre el grado de discrecionalidad que guarda el gobierno británico para consultar y determinar el grado de apertura en la consulta de documentos públicos. Debieron pasar más de diez años, y tras la caída del Muro de Berlín en 1989, para que el tema se volviera a debatir en foros académicos (Chandler, 1998). En esta época histórica se comienza a incluir la necesidad de utilizar las tecnologías de información y comunicación para impulsar la idea del gobierno transparente que promoviera la rendición de cuentas y la apertura de los datos (Butler et al., 2004; Porte et al., 2005; Welch y Wong, 2001).

Sin embargo, la idea del gobierno abierto no quedó completamente clara. Por el contrario, el ingreso de la tecnología ha hecho más complejo desarrollar dicha idea y permitir una mayor comprensión del fenómeno. En este marco se inscribe la presente investigación que busca contribuir con una conceptualización teórica del fenómeno. Para resolver dicha necesidad teórica se revisó la literatura científica sobre el tema para construir un estado del arte que posibilite dar luz a un concepto de gobierno abierto. En esta búsqueda solamente se eligieron revistas académicas internacionales que presentaran el desarrollo, discusión y debate teórico del gobierno abierto desde la primera publicación (Parks, 1957) hasta el año 2014.

Deliberadamente se omitieron publicaciones no arbitradas para darle más validez a la construcción teórica. Para su mayor comprensión, el presente 
artículo se divide en cuatro grandes secciones. La primera de ellas es esta introducción que precisa el problema de estudio. La segunda describe el marco teórico a partir del cual se genera la conceptualización de gobierno abierto, dividida en tres grandes corrientes teóricas: 1) la libertad de información, 2) la información como herramienta, y 3) datos abiertos (open data). La tercera sección presenta la solución que sería una propuesta conceptual para entender el gobierno abierto. La cuarta expone las limitaciones teóricas y los retos futuros que puede tener el gobierno abierto en el ámbito teórico.

\section{Marco teórico}

Como resultado de una extensa revisión teórica descrita en la introducción se encontraron más de 50 referencias en revistas científicas internacionales, relacionadas con aspectos teóricos -conceptualización, modelos teóricos, construcción de teoría - del gobierno abierto. Como una forma de sistematizar estos contenidos se consideró agruparlos en tres grandes direcciones teóricas: 1) libertad de información, 2) información gubernamental como herramienta, y 3 ) datos abiertos (véase la Figura 1). ${ }^{1}$ A continuación se describe cada una de ellas y el conjunto de referentes teóricos que agrupan.

\section{Primera dirección: La libertad de información}

El gobierno abierto es producto de un debate histórico por el derecho de los ciudadanos a tener acceso a la información gubernamental. El punto central que origina este debate es la democracia: pues esta libertad de información forma parte de las libertades que otorga la democracia. En este sentido, Richardson (1973) dice que la libertad de información es un componente básico del proceso democrático; de acuerdo con esta idea llamada "derecho de saber" (right to know), se convierte en lo opuesto al secreto gubernamental. En la lucha entre determinar qué se puede saber y qué es un derecho a saber es donde se enfoca el derecho de la información y las múltiples leyes reglamentarias -actas, decretos, etc.- que buscaban regularlo (Drachsler, 1976).

Uno de los autores representativos de esta tendencia es Bennett (1985), quien analiza la campaña del gobierno británico por reducir el secreto en sus archivos y propone una ley de libertad de información que ayude a la apertura de registros gubernamentales y facilite su acceso por parte de los ciudadanos.

1 Dicha figura se encuentra al final de este artículo (Nota del Editor). 
A partir de las investigaciones de Morris et al. (1981), comienza el estudio sistemático sobre la libertad de información y el debate entre el secreto gubernamental y el acceso a la información. Otras contribuciones sobre dicha libertad vienen de Perritt (1996), quien hace una revisión de las políticas públicas y las nuevas prácticas que buscaban abrir la información gubernamental; sin embargo, realizó el estudio a partir de un enfoque descriptivo y poco profundo. En cambio, Chandler (1998) se enfoca a estudiar la Agencia Central de Telecomunicación y Computación (CCTA, siglas en inglés del Reino Unido), así como el caso de estudio de cuatro sitios del gobierno local de este país, indicando que aún faltaría liberar mucha más información para que sea realmente gobierno abierto.

Otros académicos europeos llevaron a cabo investigaciones similares, tomando casos de estudio en los gobiernos locales o federales (Andersen, 2002; Bayne, 1984; Bouwman y Nouwens, 1999). No obstante, es hasta el año 2000 cuando el auge de la computación y el uso de las tecnologías de la información y comunicación comienzan a crear una nueva dinámica en este esfuerzo de libertad informativa. Roberts (2000) describe tres obstáculos para liberar la información que ocurre en el caso canadiense.

El primero es que los recortes presupuestales sobre lo "no esencial" han causado retrasos al entregar la información y cumplir las leyes de acceso a ésta; el segundo es que muchas funciones gubernamentales se delegan a contratistas privados y organizaciones no gubernamentales no cumplen con los lineamientos de acceso a la información; y tercero, finalmente el hecho de que algunos gobiernos cobren por la información, la cual debería ser gratuita, se convierte en una barrera para que los ciudadanos tengan libre acceso a ella. Años más tarde, Zuiderwijk y Janssen (2014) hacen un estudio con funcionarios holandeses para categorizar 17 barreras que impiden liberar la información gubernamental.

Otros casos que han reforzado la idea de libertad de información como derecho, por ejemplo, argumentan que los cambios en las leyes al respecto han modificado la capacidad de los ciudadanos de acceder a mayor información porque han reestructurado a la burocracia. En cambio, para Susman (2001), una de las cuatro ventajas del gobierno electrónico es precisamente que lograr un mayor intercambio de datos con los ciudadanos obligaría al gobierno a ser más cuidadoso porque éstos podrían convertirse en sus vigilantes - watchdog- y reducir así la corrupción.

Siguiendo esta idea, nuevamente Roberts (2005) argumenta en un comparativo de libertad de información entre Canadá y el Reino Unido que la bu- 
rocracia cambia sus rutinas y su organización para "minimizar el potencial" de las leyes de acceso a la información, por lo tanto la libertad de información debería considerarse más que un derecho una obligación gubernamental.

Un hecho que fue determinante para impulsar esta idea de la libertad de información y, por ende, compartir información entre las distintas dependencias, es el atentado terrorista a las Torres Gemelas en Nueva York en el año 2001. En este sentido, Uhl (2003) argumenta la interrelación que existe entre el derecho a la libertad de información o el derecho de saber sobre la infraestructura y la seguridad interna de Estados Unidos. En este país el estudio sobre la libertad de información ha tenido un largo camino desde la Segunda Guerra Mundial (Parks, 1957) y posteriormente con la experiencia del acta de libertad de información (Relyea, 1977) hasta sus avances posteriores al memorándum enviado por el presidente Barack Obama para impulsar el gobierno abierto (Relyea, 2009).

La tendencia del gobierno abierto es mundial. Una investigación sobre cuatro países escandinavos revela diferencias importantes sobre el acceso a la información donde se protege el anonimato de una fuente, así como los derechos de los periodistas y el acceso privilegiado a los registros públicos en comparación con los países occidentales (Bertil, 1983) y en la India (Kaushik, 2012).

Otro caso interesante por las implicaciones políticas e ideológicas que representa es la libertad de información en China (Zhang y Zheng, 2009; Zheng, 2009), tanto el control de internet (Zheng, 2009) como su política de apertura de información que se encuentra limitada y en control del Estado (Xiao, 2010). Una investigación de Xiao (2012) se enfoca en cómo fluye la información gubernamental en este país, su madurez y el ambiente en el cual se contextualiza la información del gobierno.

El caso de Japón es paradigmático a partir de la lucha por el derecho a la información y las fuentes periodísticas, de acuerdo con lo que reporta Beer (1971), quien demuestra las restricciones gubernamentales para el acceso a la información en este país. Esto es confirmado años más tarde por Okudaira (1983), al describir el debate existente para crear leyes que apoyen la idea de un gobierno abierto en una Constitución. Okudaira detalla este proceso en otra publicación años después y hace un comparativo de constituciones americanas y europeas con la japonesa (Okudaira, 1990).

Otros autores han estudiado el tema de la libertad de información, desde el punto de vista de: su impacto (Birkinshaw, 2010), la gobernanza de la información y el acceso a los datos (Shepherd et al., 2010), el impacto eco- 
nómico (McClean, 2010) y la medición o evaluación de la apertura gubernamental (Hazell y Worthy, 2010). Michener (2011) revisó 85 leyes acerca de la libertad de información en distintos países del mundo. Richter y Wilson (2013) han analizado distintas aproximaciones a la gobernanza de la información en los gobiernos locales a partir de la visión de los ciudadanos, lo cual sugiere, a su juicio, que aún falta mucho por desarrollar en el gobierno abierto en Inglaterra. Finalmente, Richter y Wilson (2013) han estudiado si la transparencia -o el gobierno abierto- lleva a alcanzar mayores niveles de confianza en el gobierno; estos autores sostienen que, en efecto, hay cambios en los niveles de confianza, sobre todo en los ciudadanos que tenían escaso conocimiento del gobierno y bajo nivel de confianza.

Ante este conjunto de ideas relacionadas con la libertad de información se puede argumentar que dicha tendencia teórica se ha convertido en antecedente directo de lo que hoy se denomina gobierno abierto y que se encuentra vinculado con la transparencia. La libertad de información es una piedra angular para entender las raíces que orientan la idea de un gobierno más abierto y transparente con los ciudadanos, pues esta libertad se ha ido transformando a lo largo del tiempo, según demuestran los autores previamente mencionados, hasta convertirse en un sólido cuerpo de ideas que representan esta libertad democrática en un concepto como el gobierno abierto.

\section{Segunda dirección: La información gubernamental como herramienta}

La segunda corriente teórica considera la información gubernamental como una herramienta que facilita la toma de decisiones. Parte de la idea de la libertad de datos. La demanda de información desde los ciudadanos, la relación de confianza gobierno-ciudadanos y la relación entre gobierno-apertura de información son actividades que explican la implementación de la transparencia que pueden producir los datos gubernamentales, así como la forma en que la información gubernamental se organiza o se vincula entre sí (West, Noveck, y Sirianni, 2009).

Un texto fundamental para entender esta nueva era de apertura de información gubernamental es el libro Open Government: collaboration, transparency and Participation in Practice de Lathrop y Ruma (2010), que reúne un conjunto de autores, quienes abordan el tema del gobierno abierto desde esta perspectiva práctica; sin embargo, en esta sección se presenta un recuento de los principales autores y más recientes investigaciones sobre el tema que van en esta dirección. 
Un primer estudio sobre el uso del gobierno abierto y las necesidades de información corre a cargo de Chandler (1998), quien es el primero en sugerir la idea de que la información debe ser adaptada a las necesidades de los ciudadanos. Como resultado de una investigación en cuatro sitios web de estados en el Reino Unido, Chandler (1998) sugiere también que dicha calidad de información debe llegar a las páginas web federales y supranacionales, como es el caso de la Unión Europea.

Varios académicos han seguido esta línea de pensamiento, tal es el caso de Barata y Cain (2003), quienes han propuesto cómo deberían manejarse los registros gubernamentales de los archivos nacionales de Estados Unidos para lograr abrirse de forma rápida y efectiva. Piotrowski, Zhang, Lin y Yu (2009) entrevistaron a funcionarios de nivel medio para comparar la forma de implementación de leyes del gobierno abierto en la administración pública de China con otros países.

Siguiendo esta idea, una investigación del Centro para la Tecnología en el Gobierno (СтG por sus siglas en inglés) de la Universidad de Albany en los Estados Unidos describe una serie de estrategias para promover el gobierno abierto que son evaluadas por un conjunto de oficiales públicos. Entre los hallazgos se encontraron varios retos como: que la información se ajusta a las distintas necesidades de los usuarios; ponen como ejemplo el caso de los registros de propiedad que son distintos a los tipos de registros que se requieren por otras áreas de investigación (Dawes, 2010; Helbig et al., 2010).

La investigación de Alanazi y Chatfield, (2012) analiza como se comparten datos propiedad del gobierno en países del Medio Este discute los niveles de madurez en los que se ha avanzado la apertura de gobierno en estos países, tomando como base los ocho principios del Open Data Working Group de 2007.

Otro ángulo en esta corriente de pensamiento es detectar las necesidades de información tanto de los ciudadanos como de funcionarios públicos, y abrir ésta para hacerla útil a las dos partes. Una investigación en Egipto encontró que los funcionarios de gobierno están de acuerdo en usar las redes sociales para compartir información; no obstante, requieren entrenamiento para entender aquella que publican, y destacan la necesidad de reflexionar acerca del contexto que viven los ciudadanos en ese momento para presentar la información e impulsar cualquier iniciativa de gobierno transparente (Klischewski, 2012).

Por otro lado, el análisis de la información en China resulta muy interesante, debido a que su modernización social y tecnológica ha creado crisis de 
gobernabilidad, que busca ser resuelta con la apertura de la información. El investigador Chuansheng (2007) discute los problemas que se presentan en este país - credibilidad política, barreras legales y brecha digital- para impulsar la transparencia y rendición de cuentas de la información gubernamental. Xiao (2010) describe el modelo de apertura de información de China.

Por otro lado, Curtin y Meijer (2006) proponen lo siguiente para hacer posible esta idea de información abierta: 1) La transparencia aumenta la legitimidad, 2) la transparencia produce legitimidad, y 3) genera una legitimidad social. En este estudio, los autores llegan a la conclusión de que si bien la apertura gubernamental es un insumo importante para los procesos democratizadores, no hay una fuerte evidencia que lo relacione directamente con la legitimidad. Otras investigaciones recientes comparten esta misma idea, en el sentido de que no existe una razón directa para pensar en la legitimidad y la apertura de datos (Grimmelikhuijsen, 2010; Grimmelikhuijsen, 2009; Grimmelikhuijsen y Meijer, 2014).

La vinculación de datos ha sido estudiada como uno de los usos de la información por Wood (2011) a partir de los costos-beneficios que cada dependencia debe darle a los datos que genera, así como la flexibilidad para producir éstos, escalarlos, organizarlos y guardarlos. Un ejemplo de ello es la investigación realizada en el gobierno brasileño donde se vinculan los datos y se mide el grado de implementación del gobierno transparente para lograr la apertura y rendición de cuentas (Matheus et al., 2012).

Otro elemento de esta corriente de pensamiento donde el gobierno abierto puede ser entendido como una herramienta de información es considerarlo como una fuente que genera valor público (Harrison et al., 2011). Y un último componente de dicha corriente es el que se refiere a tratar de modelar el impacto del gobierno abierto y el contexto social. Un esfuerzo en esta dirección es el realizado por Scholl y Luna-Reyes (2011), quienes a partir del uso de la dinámica de sistemas lograron proponer un modelo que combinara el uso de la información gubernamental, la participación y la colaboración de los ciudadanos.

Otros modelos intentan explicar la implantación de políticas públicas gubernamentales en el gobierno. En este sentido, Lee y Kwak (2011) han propuesto un modelo evolutivo que a través de distintas etapas intenta explicar la implementación del gobierno transparente. Un modelo similar pero basado en los datos abiertos -que se verán en el siguiente apartado- es el propuesto por Solar et al. (2012), donde presentan 33 variables críticas para analizar debilidades y fortalezas de la apertura de datos gubernamentales. 
Esta corriente de pensamiento entiende que el gobierno abierto es una herramienta que impulsa la cooperación, el debate, la construcción conjunta de ideas, la participación ciudadana y la colaboración entre el ciudadano y el gobernante en turno (Scholl y Luna-Reyes, 2011). Alentando los procesos democráticos y de rendición de cuentas, Heckmann (2011:1) dice que es "el instrumento más prometedor para repensar y acentuar el concepto de ciudadanía en una sociedad democrática”.

\section{Tercera dirección: los datos abiertos}

La tercera corriente teórica que se ha desarrollado con rapidez en los últimos años, y cuya confluencia ha impactado al gobierno abierto, es la que se refiere a la apertura de los datos como un complemento indispensable para lograr una apertura gubernamental. Si bien los datos son la expresión básica de apertura de información -ya que son implícitamente un componente de informaciónno por ello deja de ser fundamental plantear que se abran los datos y, como resultado, la información gubernamental. Además de que los datos en su formato más simple pueden ser editados, sistematizados y analizados por los ciudadanos a detalle para tener una visión más amplia sobre los asuntos públicos.

Incluso estos datos abiertos pueden impulsar la coproducción, colaboración y participación de los ciudadanos en los procesos públicos al proveer a los funcionarios públicos de nuevos insumos de información que muestren un enfoque diferente, alternativas o propuestas distintas a la visión gubernamental, convirtiéndose así en consumidores de la información gubernamental (Puron-Cid et al., 2012).

Esta dirección había sido propuesta desde la perspectiva de la Libertad de Información (primera dirección) por Relyea (1977), cuando propone la idea de vigilancia de los datos como una forma de abrir éstos. Sin embargo, a partir de 2010, el impulso del gobierno abierto crea un auge en la investigación de datos abiertos gubernamentales, que ha originado al menos tres grandes direcciones: una primera es precisamente que los datos sirven para la rendición de cuentas; una segunda es presentar métodos, mecanismos o técnicas que ayuden a la apertura de los datos y la manera como pueden ser organizadas las dependencias públicas para lograr este cometido. La tercera dirección es la apertura de los datos desde la perspectiva ciudadana como una forma de alentar la participación con el gobierno.

En relación con la primera dirección (datos que sirven para la rendición de cuentas), las investigaciones del Centro para Tecnología del Gobierno (CTG) sobre el caso de "The American Recovery and Reinvestment Act" exi- 
gían una publicación inmediata y en tiempo real de los fondos otorgados a este programa y cuyo escrutinio sería directo a través de internet (Helbig et al., 2010). El caso de la alcaldía de Nueva York que transformó su sistema de información interno para ser público y de atención ciudadana se ha convertido en un ejemplo a seguir (Newsom y Dickey, 2013).

Dos aportaciones para entender la segunda dirección de los datos abiertos y el gobierno se refieren a los casos de cómo organizar información flexible para el sector público (Hornnes et al., 2010) y al de uso de datos abiertos sobre el censo en España (Fernández et al., 2011). En cambio, la aportación de Kalampokis et al. (2011), que propone el desarrollo de guías, manuales o lineamientos que ayuden a comparar la implementación y los esfuerzos de apertura de datos en Europa, resulta relevante al ser una de las escasas contribuciones que buscan organizar este campo estructuradamente.

Por otro lado, la investigación de Peled (2011) encontró problemas en la implementación de los programas de apertura de datos y la colaboración en Estados Unidos; entre dichos problemas, se hallan los de integración de datos en los procesos como inclusión, confusión y difusión. Su investigación concluye proponiendo un mercado federal de información que incentive el intercambio de ésta entre dependencias. Otra investigación complementaria es la que se refiere al monitoreo de los datos gubernamentales (Krabina, 2012) y una más es la que busca encontrar la intención gubernamental en la publicación de datos (Sayogo y Pardo, 2012).

Anneke Zuiderwijk, Keith Jeffery y Marijn Janssen (2012) se han enfocado a investigar los obstáculos para desarrollar políticas públicas vinculadas con la apertura de datos gubernamentales, así como de la aplicación de metadatos como una forma de alentar un mayor detalle en el uso de los datos abiertos. Finalmente, estos estudiosos son de los primeros en incursionar en la investigación de datos abiertos en el poder judicial.

En materia de apertura de datos, los esfuerzos internacionales no han sido menores, como es el caso de los países del Golfo Pérsico (Elbadawi, 2012) o el europeo para compartir datos fiscales y homologarlos (Alvarez et al., 2012). El programa de desastres nacionales de Noruega para informar a los pilotos y organizar la información gubernamental en el caso de un percance nacional (Myrseth, 2012). El caso de Japón que crea opiniones sociales y preocupaciones como argumentación ideal (sociA, en inglés) y realiza una búsqueda de contenidos a través de minería de datos en forma semiestructurada, para entender las preocupaciones de los ciudadanos (Shiramatsu et al., 2012). 
Otros países han desarrollado distintas estrategias de implementación de datos abiertos como: Kenya (Mutuku y Colaco, 2012) Colombia (Prieto, 2012), Brasil (Matheus et al., 2012) Italia, que la vincula con interoperabilidad (Lodi, Maccioni y Tortorelli, 2012).

En cuanto a la tercera dirección es la forma como los ciudadanos usan los datos abiertos para resolver sus propias necesidades de información. En este sentido, las investigaciones se vinculan directamente al uso de redes sociales y datos abiertos. Por ejemplo, el proyecto somus propone diversas herramientas para abrir los datos gubernamentales y aprovecharlos (Näkki et al., 2011). O bien, el uso de la herramienta de crowdsourcing (multitudes inteligentes) para impulsar la participación de los ciudadanos (Nam, 2010).

Por otro lado, Linders (2011) propone un grupo de categorías para medir la coproducción de datos de los ciudadanos junto con el gobierno. Recientemente el ex alcalde de San Francisco publicó un conjunto de experiencias ciudadanas acerca del uso de los datos abiertos, que ha permitido mejorar los servicios gubernamentales, alentar la participación y la colaboración en aras de una mayor interacción entre ambas partes (Newsom y Dickey, 2013).

Siguiendo esta idea, el desarrollo de aplicaciones móviles (Apps) que se pueden descargar en los teléfonos inteligentes ha sido una de las maneras más atractivas para que los ciudadanos descarguen datos y los utilicen libremente (Sandoval-Almazán et al., 2012). Numerosas aplicaciones acerca del tráfico, educación, salud, impuestos han sido desarrolladas por los propios ciudadanos usando datos gubernamentales y dando acceso gratuito a diferentes catálogos de información o datos concretos que sean útiles para la toma de decisiones cotidianas (Harsh y Ichalkaranje, 2015; Milam y Avery, 2012; Sandoval-Almazán y Rojas-Romero, 2014).

Las tres corrientes teóricas que he denominado direcciones han explicado las distintas variantes que han seguido académicos, oficiales públicos y servidores públicos para entender el gobierno abierto y se resumen en la Figura $1 .^{2}$ En ellas se intentan describir distintos caminos para entender lo que ahora se llama gobierno abierto. En el siguiente apartado se describe una propuesta conceptual de gobierno abierto que si bien no necesariamente integra todas las vertientes anteriores, pretende contribuir al debate y precisar una dirección más que ayude al entendimiento de este campo de estudio.

2 Esta Figura se encuentra al final del presente artículo (Nota del Editor). 


\section{Gobierno abierto: una propuesta conceptual}

Como resultado de este marco de ideas sobre el gobierno abierto, las distintas aportaciones teóricas, propongo el siguiente concepto: el gobierno abierto debe entenderse como una plataforma tecnológica institucional que convierta los datos gubernamentales en datos abiertos para permitir su uso, protección y colaboración por parte de los ciudadanos en los procesos de decisión pública, rendición de cuentas y mejoramiento de los servicios públicos.

En esta propuesta conceptual, el gobierno abierto puede comprenderse como una plataforma tecnológica. El uso de plataformas en el gobierno no es nuevo (Bharosa et al., 2013; Charalabidis y Loukis, 2012; Hof, 2002), es el uso de un conjunto de tecnologías de información interconectadas a través de un sólo lenguaje o plataforma y permite utilizar todo el potencial de interacción e interconexión que posibilitan las plataformas.

No sólo el uso de internet a través de los sitios web, sino de las plataformas sociales tales como Facebook y Twitter (Dijck, 2013) y del uso de tecnología móvil para difundir e interactuar con la información (Lorenzi et al., 2014).

Al ser el gobierno abierto, desde su origen, una "plataforma", puede colocarse en ella un conjunto de programas, herramientas teóricas y prácticas que faciliten la libertad de la información gubernamental. No obstante, esto no podría lograrse si no se tiene el apoyo legal y presupuestal necesario, por ello se considera la "institucionalización" de la plataforma que la habilite con los recursos necesarios y el impulso político conveniente para llevarse a cabo y extender la temporalidad de su acción -más allá de los periodos gubernamentales-, para lograr la apertura del gobierno.

En este sentido, ambos conceptos, tanto la plataforma como la institucionalización, son el resultado de la herencia histórica de la corriente teórica de libertad de información, donde uno de los componentes - para llevarse a cabo- siempre fue la voluntad política y la ausencia de alguna organización o plataforma que pudiera hacer posible el gobierno abierto.

Otro componente que incluye esta conceptualización es que el resultado directo de esta plataforma es "convertir los datos gubernamentales en datos abiertos". Esta idea parte del supuesto de que los datos del gobierno se encuentran en formatos y con lenguajes burocráticos poco accesibles para los ciudadanos. La finalidad de tener una plataforma con tecnología que ayude a la homologación y conversión de los datos para hacerlos accesibles y facilitar su difusión y utilidad es un imperativo que responde a la corriente teórica de datos abiertos. 
Una vez que los datos están "libres", que pueden ser compartidos, reutilizados y son fácilmente encontrables, los ciudadanos podrán usarlos, convirtiéndose así en datos útiles para la toma de decisiones, la comparación y, por supuesto, la rendición de cuentas de los servidores públicos y los procesos de la administración pública, lo cual proviene de la segunda corriente teórica que presenta los datos gubernamentales como herramienta para facilitar las decisiones.

Otra ventaja que ofrecen los datos gubernamentales una vez abiertos es permitir su protección, tanto de parte del gobierno hacia aquellos que considere delicados para la seguridad nacional o para la toma de decisiones públicas, como por parte de los ciudadanos para que no se vean corrompidos o contaminados de percepciones ideológicas o tendencias políticas que impidan su correcto uso y comparación; e incluso que puedan ser alterados con el propósito de evadir la rendición de cuentas o facilitar la impunidad de funcionarios o dirigentes políticos.

Al "liberar" los datos y hacerlos transparentes se convierten en un bien público. Mientras se encuentran encerrados en los archivos gubernamentales son propiedad privada del gobierno en turno, o de los funcionarios públicos que los administran. Por ello, para lograr un gobierno transparente en todos los sentidos es necesario impulsar la colaboración ciudadana en el manejo de los datos, lo cual es un componente de la democracia y de la participación ciudadana bajo un contexto más informado.

\section{Limitaciones y retos del concepto de gobierno abierto}

Esta propuesta teórica presenta al menos tres limitaciones que deben considerarse en el momento de la construcción del concepto y que no hemos dejado al margen de la discusión, pero que al menos, por el momento, no se pueden resolver todas conceptualmente.

La primera limitación es que un gobierno abierto no podría ocurrir sin la tecnología. La mayoría de las investigaciones, casos de implementación, ejemplos prácticos estudiados en la revisión de literatura -vistos anteriormente- mencionan el uso de la tecnología y en particular de las tecnologías de información y comunicación que se presentan a través de Internet como herramientas implícitas en la construcción de esta area de conocimiento.

Aunque Parks (1957) habría hecho válida su solicitud de información sin necesidad de la tecnología, no por ello habría sido cumplida y resuelta por la autoridad. El problema de la administración pública antes de los años no- 
venta era su incapacidad organizativa para resolver las posibles demandas de información por los costos de transacción que implicaban (tiempo, personal, búsqueda en archivos, etcétera) (Dawes, Pardo y DiCaterino, 1999).

Otro ángulo del problema fue la libertad de información. Si el gobierno no era capaz de otorgarla, al menos que permitiera el acceso a ella. Liberar la información y abrir los datos fueron los mandatos posteriores que no llegaron a cumplirse cabalmente sino hasta que el avance tecnológico y el uso de las computadoras y de las redes de tecnología permitían la organización, clasificación, resguardo y acceso a los datos gubernamentales (Kubicek, 2008).

La segunda limitante es que el gobierno transparente no es tan simple como parece a simple vista. La posibilidad de reorganizar los datos a través de nuevos y mejores procesos, de abrir repositorios gubernamentales de datos a los ciudadanos en una forma accesible, organizada y neutral sin afectar la privacidad de la información o exponer datos demasiado delicados que puedan dañar la función del Estado se convierte en una titánica tarea.

Ya lo comenta Meijer (2012), quien indica que la transparencia gubernamental no puede dejarse solamente en manos de los técnicos o de la tecnología, sino que debería administrarse un proceso complejo de decisiones para abrir la información gubernamental. El concepto propuesto no abarca esta limitación de complejidad en la implementación, más bien propone una dirección ideal hacia la cual debería alcanzarse.

La tercera limitante del concepto es que los gobiernos abiertos están emergiendo en todo el mundo con diferentes niveles de madurez y grados de implementación. No se trata de una tendencia ideológica o política de unos cuantos años, sino que obedece a una necesidad tanto de los ciudadanos como de los gobernantes para abrir los datos y compartir la información. En este sentido, una tarea extraordinaria que tiene el gobierno abierto es garantizar el acceso a la información a todos los ciudadanos. Esto implica reducir la brecha digital entre sus gobernados, lo cual requiere cambios tanto en infraestructura como en el mercado de las telecomunicaciones y los datos (Armenta et al., 2012; Bauerlein, 2011; Hossain, 2007).

Los distintos estudios de otros países exponen diferentes limitaciones, enfoques y procesos para implementar el gobierno abierto; ello implica que no tendrá el mismo impacto en países asiáticos que africanos o en países de Europa en comparación con Estados Unidos o América Latina; esta disparidad en los contextos, la forma de interpretación del concepto y las herencias históricas pueden ser determinantes para entender el impacto del gobierno 
abierto en cada región del mundo, contexto social u económico. El concepto presentado intenta establecer una categoría de análisis que ayude a interpretar estas distintas limitaciones y a establecer un punto de partida teórico sobre cómo estudiar el gobierno abierto.

Los retos a los cuales nos lleva esta aproximación conceptual son varios, pero uno de los más importantes es lograr claridad entre transparencia y gobierno abierto. Ambos conceptos se han confudido, mezclado entre sí, e incluso se han convertido en sinónimos en el discurso gubernamental.

La primera distinción que debemos hacer es hablar de transparencia y apertura. Desde la perspectiva conceptual que aquí se ha expuesto, lo primero es lograr la apertura, tanto de los datos como de los procesos. En este sentido, el concepto más amplio debe ser la apertura gubernamental que permita observar las distintas formas de transparencia, rendición de cuentas y apertura de datos.

Algunos autores como: Ivester (1977), Parks (1957) y Relyea (1977) apoyan esta idea, argumentando en esta dirección donde la apertura gubernamental es la primera etapa. Posteriormente, el más reciente promotor del gobierno abierto, el presidente norteamericano Barack Obama presenta su idea de apertura gubernamental primeramente y después la transparencia: "the open government initiative would establish a system of transparency, public participation, and collaboration” (McDermott, 2010). Donde se establece que la transparencia es un sistema junto con la participación pública y la colaboración. Es decir, la transparencia se convierte así en un medio para lograr un fin último: alcanzar el gobierno abierto.

Para Lathrop y Ruma (2010) consideran que es más importante generar la transparencia en primer lugar para lograr un gobierno abierto, en cambio los que piensan que el gobierno abierto es la primera etapa que debe desarrollarse son O’Reilly (2010) y Obama (2009).

Otro enfoque para entender esta relación entre transparencia y gobierno abierto sería ver la transparencia como una acción. Transparencia es el efecto de la apertura. Cuando nos referimos a "abrir el gobierno", estamos hablando de transparentar los procesos, los datos, los archivos, la información gubernamental que ha estado resguardada. La apertura es la dirección, la transparencia es el camino.

Linders y Wilson (2011) hacen precisamente esta aportación al contenido de la iniciativa de gobierno abierto en Estados Unidos y colocan la discusión en el sentido de que la transparencia puede convertirse en una herramienta clave para abrir el gobierno. Finalmente, una idea paralela que 
complementa la noción de la transparencia es la de ser protectores de la información o cuidadores de la misma (stewardship, en inglés).

Dawes (2010) argumenta que tanto la "protección" como la utilidad de la información deben ser las bases que orienten la transparencia de los datos. Siguiendo su argumentación, la calidad de los datos y los retos en la medición de transparencia son determinantes para señalar el desempeño en la apertura gubernamental. El reto de distanciarse de la idea de transparencia y darle su ubicación y justa dimensión es una importante tarea para los estudiosos del gobierno abierto en el corto plazo.

Otros retos menores pero no menos importantes serán: la medición de los gobiernos abiertos y su eficiencia al liberar la información; la regulación del gobierno abierto en cuanto a formas de operación, compromisos y resultados esperados, entre algunos otros. Sin duda, seguiremos perfeccionando nuestra percepción sobre el gobierno abierto, la transparencia y la rendición de cuentas a lo largo del tiempo. Estos conceptos no pueden ser inmutables, pero al menos podrán ayudar a comprender mejor los esfuerzos públicos en este campo.

\section{Bibliografía}

Álvarez,J. M. et al. (2012), “Towards a Pan-European E-Procurement Platform to Aggregate, Publish and Search Public Procurement Notices Powered by Linked Open Data: The Moldeas Approach", en International Journal of Software Engineering and Knowledge Engineering, vol. 22, núm. 3, Londres, Inglaterra: World Scientific.

Andersen, Kim Viborg (2002), "Public Sector Process Rebuilding Using Information Systems", en Electronic Government, vol. 2456, Alemania: Springer.

Armenta, Álvaro et al. (2012), "The new digital divide: the confluence of broadband penetration, sustainable development, technology adoption and community participation", en Information Technology for Development, vol. 18, núm. 4. London, United Kingdom: Taylor y Francis.

Barata, Kimberly y Piers Cain (2003), "Records management toolkits from across the pond", en Information Management Journal, vol. 37, núm. 4, Amsterdam, Holanda.

Bayne, Peter (1984), "Freedom of Information”, en Legal Service Bulletin, vol. 9, Oklahoma, USA: NACM Mid America.

Beer, L. W. (1971), "Freedom of Information and the Evidentiary Use of Film in Japan: Law and Sociopolitics in an East Asian Democracy", en The American Political Science Review, vol. 65, núm. 4, Washington, USA: ASPA.

Bennett, Colin (1985), "From the Dark to the Light: The Open Government Debate in Britain", en Journal of Public Policy, vol. 5, núm. 2, London, United Kingdom: Taylor y Francis. 
Rodrigo Sandoval-Almazán. Gobierno abierto y transparencia: construyendo un marco conceptual

Bertil Wennergren, Hon (1983), "Freedom of information in nordic countries: Recent developments", en Government Publications Review, vol. 10, núm. 1, Amsterdam, Holanda: Elsevier

Bharosa, Nitesh et al. (2013), "Developing Multi-sided Platforms for Public-Private Information Sharing: Design Observations from Two Case Studies", en ACM, Proceedings of the 14th Annual International Conference on Digital Government Research, Quebec, Canadá: ACM.

Birkinshaw, P. (2010), "Freedom of information and its impact in the United Kingdom”, en Government Information Quarterly, vol. 27, núm. 4, Amsterdam, Holanda: Elsevier.

Bouwman, Harry y John Nouwens (1999), "Governments as electronic publishers? The Dutch case", en Government Information Quarterly, vol. 16, núm. 1, Amsterdam, Holanda: Elsevier.

Butler, Tom et al. (2004), "Promoting Knowledge Sharing in Government and NonGovernment Organizations Using Open Source Software: The pKaDs Story”, en Electronic Journal of E-Government, vol. 2, núm. 2, Kidmore End, United Kingdom: Academic Publishing Limited.

Chandler, Helen (1998), “Towards open government: official information on the Web”, en New Library World, vol. 99, núm. 6, Bingley, United Kingdom: Emerald Group Publishing Limited.

Charalabidis, Yannis y Loukis Euripidis (2012), "Participative Public Policy Making Through Multiple Social Media Platforms Utilization”, en International Journal of Electronic Government Research, vol. 8, núm. 3, Hershey, Pensilvania, USA: IGI Global.

Chuansheng, R. (2007), "E-Government Construction and China's Administrative Litigation Act”, en A.-V. Anttiroiko y M. Mälkiä [eds], Encyclopedia of digital government, Hershey, Pennsylvania: Idea Group Reference.

Curtin, Deirdre y Albert Jacob Meijer (2006), “Does transparency strengthen legitimacy?”, en Information Polity, vol. 11, núm. 2, Amsterdam, Holanda: IOs Press.

Dawes, Sharon (2010), “Stewardship and usefulness: Policy principles for information-based transparency”, en Government Information Quarterly, vol. 27, núm. 4, Amsterdam, Holanda: Elsevier.

Dawes, Sharon, Pardo, Theresa y Ann DiCaterino (1999), "Crossing the Threshold: Practical Foundations for Government Services on the World Wide Web”, en Journal of the American Society for Information Science, vol. 50, núm. 4, New York, USA: ASIS\&T.

Dijck, José van (2013), "Facebook and the engineering of connectivity A multi-layered approach to social media platforms", en Convergence: The International Journal of Research into New Media Technologies, vol. 19, núm. 2, New York, USA: Sage.

Drachsler, D. A. (1976), "Freedom of Information Act and the Right of Non-Disclosure", Administrative Law Review, vol. 28, Washington, USA: American University Washington College of Law.

Elbadawi, I. A. (2012), “The State of Open Government Data in GCC Countries”, en 12th European Conference on eGovernment (ECEG 2012), Barcelona, España.

Grimmelikhuijsen, Stephan (2009), “Do transparent government agencies strengthen trust?", en Information Polity, vol. 14, núm. 3, Amsterdam, Holanda: IOs Press. 
Grimmelikhuijsen, Stephan (2010), “Transparency of Public Decision-Making: Towards Trust in Local Government?”, en Policy y Internet, vol. 2, núm. 1, Washington, UsA: Pso.

Grimmelikhuijsen, Stephan y Albert Meijer (2014), "Effects of Transparency on the Perceived Trustworthiness of a Government Organization: Evidence from an Online Experiment", en Journal of Public Administration Research and Theory, vol. 24, núm. 1, Oxford, United Kingdom: Oxford University Press.

Harrison, T. M. et al. (2011), "Open government and e-government: democratic challenges from a public value perspective", en 12th Annual International Conference on Digital Government Research (dg.o 2011), dg.o, vol. 11, College Park, Maryland, USA: ACM.

Hazell, Robert y Ben Worthy (2010), "Assessing the performance of freedom of information", en Government Information Quarterly, vol. 27, núm. 4, Amsterdam, Holanda: Elsevier.

Heckmann, D. (2011), "Open Government - Retooling Democracy for the 21st Century", en 44th Hawai International Conference on System Sciences (HICSS-44), Kauai, Hawai, USA: IEEe, Computer Society Conference Publishing Services.

Helbig, Natalie et al. (2010), Information and transparency: learning from recovery act reporting experiences, California, USA: Digital Government Society of North America.

Hof, Sonja (2002), "Arguments for a Holistic and Open Approach to Secure e-Government", en Electronic government: First international conference, EGOV 2002, Aix-en-Provence, France, September 2-6, 2002: proceedings, vol. 2456, Berlín, Heidelberg, Germany: Springer.

Hornnes, Erick, Jansen, Arild y Oivind Langeland (2010), "How to develop an open and flexible information infrastructure for the public sector?”, en M. A. Wimmer et al. [eds.], Electronic Government: 9th IFIP WG 8.5 International Conference, EGOV 2010, Lausanne, Switzerland, Augtust/September 2010: Proceedings, Berlín, Heidelberg, Alemania: Springer-Verlag.

Hossain, Farhad (2007), "Digital Divides and Grassroots-Based E-Government in Developing Countries”, en Encyclopedia of digital government, vol. 1, Hershey, Pennsylvania, UsA: Idea Group Reference.

Ivester, Daniel Mitchell (1977), “The Constitutional Right to Know”, en Hastings Constitutional Law Quarterly, vol. 4, San Francisco, California, USA: McAllister.

Kalampokis, Evangelos, Hausenblas, Michael y Konstantinos Tarabanis (2011), "Combining Social and Government Open Data for Participatory Decision-Making", en E. Tambouris, A. Macintosh y H. Bruijn [eds.], Electronic Participation, vol. 6847, Berlín, Heidelberg, Alemania: Springer.

Kaushik, Anjali (2012), "eGovernance and Freedom of Information Act: The Indian Experience", en 12th European Conference on eGovernment (ECEG 2012), Barcelona, España: Institute of Public Governance and Management.

Klischewski, R. (2012), “Identifying Informational Needs for Open Government: The Case of Egypt", en 45th Hawai International Conference on System Sciences (HICSS- 45), Maui, Hawai, USA: IEEE.

Krabina, B. (2012), "Internal data monitoring for Open Government", en 2nd Conference for E-Democracy and Open Government (CeDEM 2012), Krems, Austria: DanubeUniversity Krems. 
Rodrigo Sandoval-Almazán. Gobierno abierto y transparencia: construyendo un marco conceptual

Kubicek, Herbert (2008), "Next Generation FoI Between Information Management and Web 2.0", en Proceeding dg.o '08 Proceedings of the 2008 International Conference on Digital Government Research, California, USA: DGSNA.

Lathrop, Daniel y Laurel Ruma (2010), Open Government: collaboration, transparency and Participation in Practice, California, UsA: O'Reilly.

Lee, G. y Y. H. Kwak (2011), “Open government implementation model: a stage model for achieving increased public engagement”, en 12th Annual International Conference on Digital Government Research (dg.o 2011), dg.o, vol. 11, Maryland, USA: ACM.

Lodi, G., Maccioni, A. y F. Tortorelli (2012), "Linked open data in the italian e-government interoperability framework", en 6th International Conference on Methodologies, Technologies and Tools Enabling e-Government (меттеG 12), Belgrade, Serbia.

Lorenzi, David et al. (2014), "Enhancing the government service experience through QR codes on mobile platforms", en Government Information Quarterly, vol. 31, núm. 1, Amsterdam, Holanda: Elsevier.

Matheus, Ricardo, Maia Ribeiro, Manuella y José Carlos Vaz (2012), “New Perspectives, for Electronic Government in Brazil: The Adoption of Open Government Data in National and Subnational Governments of Brazil", en 6th International Conference On Theory And Practice Of Electronic Governance, Albany, New York, UsA: Association for Computing Machinery.

McClean, T. (2010), "Who pays the piper? The political economy offreedom of information", en Government Information Quarterly, vol. 27, núm. 4, Amsterdam, Holanda: Elsevier.

McDermott, P. (2010), “Building open government", en Government Information Quarterly, vol. 27, núm. 4, Amsterdam, Holanda: Elsevier.

Meijer, Albert et al. (2012), "Communities of Public Service Support Citizens engage in social learning in peer-to-peer networks", en Government Information Quarterly, vol. 29, núm. 1, Amsterdam, Holanda: Elsevier.

Michener, Greg (2011), “For Laws Around the World”, en Journal of Democracy, vol. 22, núm. 2, Washington, DC, United States of America: Project Muse.

Milam, Lacey y Elizabeth Johnson Avery (2012), "Apps4Africa: A new State Department public diplomacy initiative”, en Public Relations Review, vol. 38, núm. 2, Amsterdam, Holanda: Elsevier.

Morris, Robert et al. (1981), "Research and the Freedom of Information Act", en American Psychologist, vol. 36, núm. 8, Washington, United States of America: American Psychological Association.

Mutuku, Leonida y Jessica Colaco (2012), "Increasing Kenyan Open Data Consumption: A Design Thinking Approach", en 6TH International Conference on Theory and Practice of Electronic Governance, Albany, New York, USA: Association for Computing Machinery.

Myrseth, P. (2012), "National Master Data As 5 Star Linked Open Data”, en H. J. Scholl et al. [eds], Electronic Government and Electronic Participation: Joint Proceedings of Ongoing Research and Projects of IFIP EGOV and IFIP ePart 2012, Viena, Austria: Trauner Verlag.

Newsom, Gavin y Lisa Dickey (2013), Citizenville: How to Take the Town Square Digital and Reinvent Government, Westminster, London, United Kingdom: Penguin Press HC. 
Convergencia Revista de Ciencias Sociales, núm. 68, 2015, Universidad Autónoma del Estado de México

Okudaira, Y. (1983), “Toward freedom of information: The Japanese case”, en Government Publications Review, vol. 10, núm. 1, Amsterdam, Holanda: Elsevier.

Okudaira, Y. (1990), "Forty Years of the Constitution and Its Various Influences: Japanese, American and European", en Law and Contemporary Problems, vol. 53, núm. 1, Durham, North Carolina, USA: Duke University School of Law.

O'Reilly, T. (2010), “Government as a platform”, en Open Government: Collaboration, Transparency and Participation in Practice, Los Angeles, California, UsA: O'Reilly Media.

Parks, Wallace (1957), “The Open Government Principle: Applying the Right to Know under the constitution", en The George Washington Law Review, vol. 26, núm. 1, Washington, USA: The George Washington University Law School.

Peled, A. (2011), "When transparency and collaboration collide: The UsA Open Data program", en Journal of the American Society for Information Science and Technology, Journal of the American Society for Information Science and Technology, vol. 62, núm. 11, Silver Spring, Maryland, USA: ASIS\&T.

Perritt, Jr. Henry (1996), “The information highway: On ramps, checkpoints, and tollbooths”, en Government Information Quarterly, vol. 13, núm. 2, Amsterdam, Holanda: Elsevier.

Piotrowski, Suzanne et al. (2009), "Key Issues for Implementation of Chinese Open Government Information Regulations”, en Public Administration Review, vol. 69, núm. S1, Washington, USA: ASPA.

Porte, Todd La et al. (2005), "Governance in the Era of the World Wide Web: An Assessment of Organizational Openness and Government Effectiveness, 1997-2001”, en G. D. Garson [ed.], Public Administration and Public Policy: A Comprehensive Publication Program, vol. 111, Boca Raton, USA: Taylor y Francis, IGI global.

Prieto, Lydia Marleny (2012), "Implementation Framework for Open Data in Colombia", en 6th International Conference On Theory And Practice Of Electronic Governance, Albany, New York, UsA: Association for Computing Machinery.

Puron-Cid, Gabriel et al. (2012), "IT-enabled policy analysis: new technologies, sophisticated analysis and open data for better government decisions", en 13th Annual International Conference on Digital Government Research (dg.o '12), Maryland, USA: ACM.

Relyea, Harold (1977), “The provision of government information: the federal Freedom of Information Act experience", en Canadian Public Administration, vol. 20, núm. 2, Toronto, Canadá: IAPC.

Relyea, Harold (2009), "Federal freedom of information policy: Highlights of recent developments", en Government Information Quarterly, vol. 26, núm. 2, Amsterdam, Holanda: Elsevier.

Richardson, E. L. (1973), "Freedom of Information”, en Loyola Law Review, vol. 20, Washington, USA: AJCU.

Richter, Paul y Rob Wilson (2013), “It"s the tip of the iceberg': the hidden tensions between theory, policy and practice in the management of Freedom of Information in English local government bodies-evidence from a regional study", en Public Money y Management, vol. 33, núm. 3, London, United Kingdom: Taylor y Francis. 
Rodrigo Sandoval-Almazán. Gobierno abierto y transparencia: construyendo un marco conceptual

Roberts, Alasdair (2000), "Less Government, More Secrecy: Reinvention and the Weakening of Freedom of Information Law", en Public Administration Review, vol. 60, núm. 4, Washington, USA: ASPA.

Roberts, Alasdair (2005), "Spin Control and Freedom of Information: Lessons for the United Kingdom from Canada”, en Public Administration, vol. 83, núm. 1, Washington, USA: ASPA.

Sandoval-Almazán, Rodrigo et al. (2012), "Open government 2.0: citizen empowerment through open data, web and mobile apps", en Proceedings of the 6th International Conference on Theory and Practice of Electronic Governance, New York, United States of America: ACM.

Sayogo, D. S. y T. A. Pardo (2012), "Exploring the Motive for Data Publication in Open Data Initiative: Linking Intention to Action", en 45th Hawaii International Conference on System Sciences (HICSS-45), Maui, USA: IEEE.

Shepherd, Elizabeth, Stevenson, Alice y Andrew Flinn (2010), "Information governance, records management, and freedom of information: A study of local government authorities in England", en Government Information Quarterly, vol. 27, núm. 4, Amsterdam, Holanda: Elsevier.

Shiramatsu, S. et al. (2012), "Structuring Japanese Regional Information Gathered from the Web as Linked Open Data for Use in Concern Assessment”, en E. Tambouris, A. Macintosh y Ø. Sæbø [eds.], Electronic Participation. Lecture Notes in Computer Science, Berlín, Heidelberg, Alemania: Springer.

Solar, Mauricio, Concha, Gastón y Luis Meijueiro (2012), "A Model to Assess Open Government Data in Public Agencies", en H. J. Scholl et al. [eds.], Electronic Government, vol. 7443, Berlin, Heidelberg, Alemania: Springer.

Susman, Thomas (2001), “The good, the bad, and the ugly: E-government and the people's right to know", en Vital Speeches of the Day, vol. 68, núm. 2, New York, United States of America: McMurry.

Uhl, K. E. (2003), "Freedom of Information Act Post-9/11: Balancing the Public's Right to Know, Critical Infrastructure Protection and Homeland Security”, en American University Law Review, vol. 53, Washington, USA: American University Washington College of Law.

Welch, Eric y Wilson Wong (2001), “Global information technology pressure and government accountability: the mediating effect of domestic context on website openness", en Journal of Public Administration Research and Theory, vol. 11, núm. 4, Oxford, United Kingdom: Oxford University Press.

West, D., Noveck, B. S. y C. Sirianni (2009), Innovation in Government: How to Make the Public Sector Faster, Smarter and More Connected, Washington, USA: Brookings Institution.

Wood, David (2011), Linking Government Data, Berlin, Heidelberg: Springer: Springer.

Xiao, Weibing (2010), “China's limited push model of For legislation”, en Government Information Quarterly, vol. 27, núm. 4, Amsterdam, Holanda: Elsevier.

Xiao, Weibing (2012), Freedom of information reform in China: Information flow analysis, Abingdon, United Kingdom: Routledge. 
Convergencia Revista de Ciencias Sociales, núm. 68, 2015, Universidad Autónoma del Estado de México

Zhang, Xiaoling y Yongnian, Zheng (2009), China's Information and Communications Technology Revolution: Political Impacts and State Responses, London, United Kingdom: Routledge.

Zheng, Yongnian (2009), “The Political Cost of Information Control in China: The Nation-State and Governance", en X. Zhang y Y. Zheng [eds.], China's information and communications technology revolution: social changes and state responses, London, United Kingdom: Routledge.

Zuiderwijk, Anneke y Marijn Janssen (2014), “The Negative Effects of Open Government Data - Investigating the Dark Side of Open Data”, en Proceedings of the 15th Annual International Conference on Digital Government Research, New York, USA: ACM.

Zuiderwijk, Anneke, Jeffery Keith y Marijn Janssen (2012), “The necessity of metadata for linked open data and its contribution to policy analyses", en 2nd Conference for E-Democracy and Open Government (CeDEM 2012), Krems an der Donau, Austria: Donau-Universität Krems.

\section{Recursos electrónicos}

Alanazi, J. y A. Chatfield (2012), "Sharing Government-Owned Data with the Public: A Cross-Country Analysis of Open Data Practice in the Middle East", en amcis 2012 Proceedings, California, USA: AISeL. Disponible en: http://aisel.aisnet.org/ amcis2012/proceedings/EGovernment/16 [9 de abril de 2015].

Bauerlein, Mark (2011), The digital divide arguments for and against Facebook, Google, texting, and the age of social networking, New York, United States of America: Jeremy P. Tarcher/Penguin. Disponible en: http://www.amazon.com/The-Digital-DivideArguments-Facebook/dp/1585428868 [14 de enero de 2015].

Fernández, Javier, Martínez-Prieto, Miguel y Claudio Gutiérrez (2011), "Publishing open statistical data: the Spanish census", en Proceedings of the 12th Annual International Digital Government Research Conference (dg.o 2011), College Park, MD, USA: ACM. Disponible en: http://doi.acm.org/10.1145/2037556.2037560 [25 de marzo de 2015].

Harsh, Akhilesh y Nikhil Ichalkaranje (2015), “Transforming e-Government to Smart Government: A South Australian Perspective”, en L. C. Jain, S. Patnaiky N. Ichalkaranje [eds.], Intelligent Computing, Communication and Devices. Germany: Springer India. Disponible en: http://link.springer.com/chapter/10.1007/978-81-322-2012-1_2 [25 de marzo de 2015].

Linders, Dennis (2011), "We-Government: an anatomy of citizen coproduction in the information age", en Proceedings of the 12th Annual International Digital Government Research Conference (dg.o 2011), College Park, Maryland, USA: ACM. Disponible en: http://doi.acm.org/10.1145/2037556.2037581 [14 de enero de 2015].

Linders, Dennis y Susan Copeland Wilson (2011), "What is open government?: one year after the directive", en Proceedings of the 12th Annual International Digital Government Research Conference (dg.o 2011), College Park, Maryland, United States of America: 
Rodrigo Sandoval-Almazán. Gobierno abierto y transparencia: construyendo un marco conceptual

ACM. Disponible en: http://doi.acm.org/10.1145/2037556.2037599 [26 de marzo de 2015].

Näkki, Pirjo et al. (2011), Social media for citizen participation Report on the Somus project, Espoo. Disponible en: http://nordicom.statsbiblioteket.dk/ncom/en/ publications/social-media-for-citizen-participation\%28e7d1a1f0-9271-11e0-b718000ea68e967b\%29.html [26 de marzo de 2015].

Nam, Taewoo (2010), “The Wisdom of Crowds in Government 2.0: Information Paradigm Evolution toward Wiki-Government”, en amcis 2010 Proceedings, Lima, Perú: AIs. Disponible en: http://aisel.aisnet.org/amcis2010/337 [26 de marzo de 2015].

Obama, Barack (2009), “Transparency and Open Government | The White House”, White House, United States of America. Disponible en: http://www.whitehouse.gov/the press_office/TransparencyandOpenGovernment/ [26 de marzo de 2015].

Sandoval-Almazán, Rodrigo y Yaneileth Rojas-Romero (2014), “The Case of the Mexican Mobile Government: Measurement and Examples”, en Zaigham Mahmood [ed.], Emerging Mobile and Web 2.0 Technologies for Connected E-Government, Hershey, Pennsylvania, United States of America: IGI Global. Disponible en: http://services. igi-global.com/resolvedoi/resolve.aspx?doi=10.4018/978-1-4666-6082-3.ch009 [26 de marzo de 2015].

Scholl, Hans Jochen y Luis Felipe Luna-Reyes (2011), “Transparency and openness in government: a system dynamics perspective", en Proceedings of the 5th International Conference on Theory and Practice of Electronic Governance, Tallinn, Estonia: ACM. Disponible en: http://doi.acm.org/10.1145/2072069.2072088 [26 de marzo de 2015].

Tauberer, Joshua (2012), Open Government Data, Amazon: Amazon Digital Services. Disponible en: http://www.amazon.com/Open-Government-Data-ebook/ $\mathrm{dp} /$ B007U69DKU/ref=sr_1_2 ? s=booksyie=UTF8yqid $=1361379716 \mathrm{ysr}=1$ 2 ykeywords=open+government $[26$ de marzo de 2015]. 
Anexo

\section{Figura 1}

\section{Direcciones teóricas del gobierno transparente}

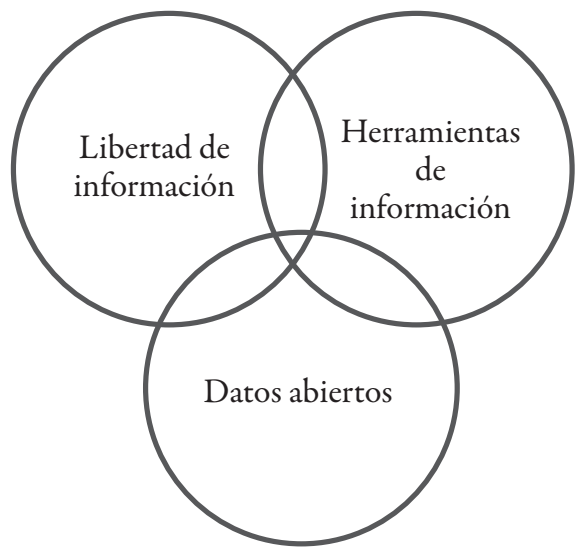

Fuente: Elaboración propia.

Rodrigo Sandoval Almazán. Profesor de la Facultad de Ciencias Políticas y Sociales de la Universidad Autónoma del Estado de México (UAEM), en la ciudad de Toluca. Ha sido profesor de la Escuela de Graduados en Administración Pública (EGAP) y de Administración de Empresas (EGADE) del Instituto Tecnológico y de Estudios Superiores de Monterrey, Campus Estado de México. Es miembro del Sistema Nacional de Investigadores Nivel 2. Líneas de investigación: gobierno electrónico, tecnologías de la información de las organizaciones, redes sociales en el gobierno y brecha digital. Publicaciones recientes: Sandoval Almazán, R. y J. R. Gil García, "Social Media in State Governments: Preliminary Results About the Use of Facebook and Twitter in México", en E-Government Implementation and Practice in Developing Countries, vol. 1, USA: IGI Global. Disponible en: http://books.google.com. $\mathrm{mx} /$ books ?hl=esylr $=$ yid $=$ bPOIWfJWFowCyoi $=$ fndypg $=$ PA128ydq $=\% 2$ 2 Rodrigo + Sandoval-Almazan $\% 22$ yots $=u$ ABrlId0fRysig $=$ HRi0BtKTheT 4OWUfixr3K2T8-O0yredir_esc=y\#v=onepageyqyf=false (2013); Sandoval-Almazán, R., La larga marcha del Gobierno Abierto. Teoría, medición y futuro, INAP, (2013a); Sandoval-Almazán, R., "Open Government Success Factors in Government Websites: The Mexican Experience", en E-Govern- 
ment Success Factors and Measures: Theories, Concepts, and Methodologies, vol. 1, USA: IGI Global. Disponible en: http://www.igi-global.com/book/ government-success-factors-measures/73563 (2013b); Sandoval-Almazán, R. y R. Gil-García, "Cyberactivism through Social Media: Twitter, YouTube, and the Mexican Political Movement «I'm Number 132»”, en Proceedings of the 46th Annual Hawai International Conference on System Sciences, vol. 1. Disponible en: http://www.cide.edu/publicaciones/status/dts/DTAP\%20 273.pdf (2013).

Recepción: 27 de agosto de 2013.

Aprobación: 15 de enero de 2015. 
